\title{
The central black hole masses and Doppler factors of the $\gamma$-ray loud blazars
}

\author{
J.H. Fan $^{1,2}$, G.Z. Xie ${ }^{3}$, and R. Bacon ${ }^{1}$ \\ 1 CRAL Observatoire de Lyon, 9 avenue Charles André, 69563 Saint-Genis-laval Cedex, France \\ 2 Center for Astrophysics, Guangzhou Normal University, Guangzhou 510400, China \\ e-mail:jhfan@guangztc.edu.cn \\ 3 Yunnan Observatory, Chinese Academy of Sciences, Kunming 650011, China
}

Received June 30; accepted October 7, 1998

\begin{abstract}
In this paper, the central black hole masses and the Doppler factors are derived for PKS $0528+134$, PKS 0537-441, 3C 279, PKS 1406-074, PKS 1622-297, $\mathrm{Q} 1633+382$, Mkn 501, and BL Lacertae. The masses obtained are in the range of $(1-7) 10^{7} M_{\odot}$ and compared with that obtained with the Klein-Nishina cross section considered (Dermer \& Gehrels 1995). If we considered only the Thomson cross section, the masses are in the range of $2.610^{6} M_{\odot}-210^{11} M_{\odot}$. The masses obtained from our method are less sensitive to the flux than those obtained from Dermer \& Gehrels (1995) method. The masses obtained from two flares (1991 and 1996 flares) of 3C 279 are almost the same. For 3C 279 and BL Lacertae, viewing angle, $\theta$, and Lorentz factor, $\Gamma$, are estimated from the derived Doppler factor and the measured superluminal velocity. For $3 \mathrm{C} 279, \theta=10$.9-15.6, $\Gamma=2.4-14.4$ for $\delta=3.37 ; \theta=8.45-9.7, \Gamma=2.95-11.20$ for $\delta=4.89$; for BL Lacertae, $\theta=25^{\circ}$. $-29^{\circ} 4, \Gamma=2.0-4.0$.
\end{abstract}

Key words: black holes - BL Lacertae objects - galaxies: jets

\section{Introduction}

One of the most important results of the CGRO/EGRET instrument in the field of extragalactic astronomy is the discovery that blazars (i.e., flat-spectrum radio quasars(FSRQs) and BL Lac objects) emit most of their bolometric luminosity in the high $\gamma$-rays $(E>100 \mathrm{MeV})$ energy range. Many of the $\gamma$-ray emitters are also superluminal radio sources (von Montigny et al. 1995). The common properties of these EGRET-detected AGNs are the following: the $\gamma$-ray flux is dominant over the flux in lower energy bands; The $\gamma$-ray luminosity above $100 \mathrm{MeV}$ ranges from less than $310^{44} \mathrm{erg} \mathrm{s}^{-1}$ to more than $10^{49} \mathrm{erg} \mathrm{s}^{-1}$ (assuming isotropic emission); many of the sources are strongly variable in the $\gamma$-ray band on timescales from days to months (Mukherjee et al. 1997), but large flux variability on short timescales of $<1$ day is also detected (see below). Some correlations between $\gamma$-ray and the lower energetic bands are discussed (see Dondi \& Ghisellini 1995; Fan 1997; Fan et al. 1998a; Mücke et al. 1997; Xie et al. 1997; Zhou et al. 1997). These suggest that the $\gamma$-ray emission is likely from the jet.

Various models for $\gamma$-ray emission have been proposed: Namely, (1) the inverse Compton process on the external photons $(E C S)$, in which the soft photons are directly from a nearby accretion disk (Dermer et al. 1992; Coppi et al. 1993) or from disk radiation reprocessed in some region of AGNs (e.g. broad emission line region) (Sikora et al. 1994; Blandford \& Levinson 1995); (2) the synchrotron self-Compton model $(S S C)$, in which the soft photons originate as synchrotron emission in the jet (Maraschi et al. 1992; Bloom \& Marscher 1992, 1993; Zdziarski \& Krolik 1993; Bloom \& Marscher 1996; Marscher \& Travis 1996); (3) synchrotron emission from ultrarelativistic electrons and positrons produced in a proton-induced cascade $(P I C)$ (Mannheim \& Biermann 1992; Mannheim 1993; Cheng \& Ding 1994). TeV radiations are observed from 3 X-ray-selected BL Lacertae objects (XBLs): Mkn 421 (Punch et al. 1992); Mkn 501 (Quinn et al. 1996), and IES 2344+514 (Catanese et al. 1998). But there is no consensus yet on the dominant emission process (see 3C 273 for instance, von Montigny et al. 1997); for PKS 0528+134, the lower and higher states can be fitted by different models (e.g. Böttcher \& Collmar 1998).

The $\gamma$-rays are produced at a distance of $\sim 100 R_{\mathrm{g}}$ (Hartman et al. 1996), $205 R_{\mathrm{g}}$ (Xie et al. 1998), and hundreds of Schwarzshild radii (Ghisellini \& Madau 1996; Celotti \& Ghisellini 1998). We think that this distance is an important parameter, which can be used to 
constrain the mass of the central black hole. In this paper, we will use it to derive the central black hole mass and the Doppler factor for some blazars with short timescales. The paper is arranged as follows: in Sect. 2, we estimate the mass of the central black hole and the Doppler factor; in Sect. 3, we give some discussions and a brief summary.

$H_{0}=75 \mathrm{~km} \mathrm{~s}^{-1} \mathrm{Mpc}^{-1}$, and $q_{0}=0.5$ are adopted through out the paper.

\section{The mass of the central black hole and the Doppler factor}

\subsection{Data}

Some $\gamma$-ray loud blazars have been observed several times with EGRET while three XBLs have been observed to show $\mathrm{TeV}$ radiations. In the following section, we present the $\gamma$-ray loud blazars with available $\gamma$-ray variation timescales. Because the variability timescale corresponds to different variation amplitude for different source and/or different observation period, we use the doubling timescale, $\Delta T_{\mathrm{D}}=\left(F_{\text {initial }} / \Delta F\right) \Delta T$, as the variability timescale.

\subsubsection{PKS 0528+134}

PKS 0528+134, $z=2.07$ (Hunter et al. 1993), is one of the most luminous examples of blazars. It is observed by EGRET, COMPTE and OSSE aboard the CGRO (see Hunter et al. 1993; McNaron-Brown et al. 1995; Mukherjee et al. 1996; Collmar et al. 1997; Sambruna et al. 1997).

During the period of 16-30 May 1991, the source showed $F(>100 \mathrm{MeV})=(1.0 \pm 0.2) 10^{-6}$ photon $\mathrm{cm}^{-2}$ $\mathrm{s}^{-1}$ with photon spectral index $\alpha_{\gamma}=2.56 \pm 0.09$.

During 23-29 March 1993, $F(>100 \quad \mathrm{MeV})=$ $(0.23 \pm 0.12-3.08 \pm 0.35) 10^{-6}$ photon $\mathrm{cm}^{-2} \mathrm{~s}^{-1}$; with a photon spectral index $\alpha_{\gamma}=2.21 \pm 0.10$. In the 1993 observation, a variation of order $100 \%$ over a timescale of $\sim 2$ days was detected (see Wagner et al. 1997), which suggests a doubling time scale of $\Delta T_{\mathrm{D}}=$ 1 day.

During August, 1994, $F(>100 \mathrm{MeV})=(0.32 \pm 0.1)$ $10^{-6}$ photon $\mathrm{cm}^{-2} \mathrm{~s}^{-1}$; with a photon spectral index $\alpha_{\gamma}=2.70$.

There is a clear evidence that the spectrum becomes harder when the $\gamma$-ray flux increases.

\subsubsection{PKS $0537-441$}

PKS $0537-441, z=0.896$, a candidate of gravitational lens (Surpi et al. 1996), is a violently variable object (Fan $\& \operatorname{Lin} 1998)$. The $\gamma$-ray flux varies from $(1.83 \pm 0.91)$ to $(8.98 \pm 1.45) 10^{-6}$ photon $\mathrm{cm}^{-2} \mathrm{~s}^{-1}$ (Mukherjee et al. 1997). A flare of a factor of $\sim 3$ from 0.35 to $2.010^{-6}$ photon $\mathrm{cm}^{-2} \mathrm{~s}^{-1}$ over a time scale of $\sim 2$ days can be seen from Fig. 3 in Hartman's paper (Hartman 1996). $\Delta T_{\mathrm{D}}=16 \mathrm{hr}$.

\subsection{3. $1253-055,3 \mathrm{C} 279$}

3C 279 is a well known member of OVV subclass of blazars. it is perhaps the prototypical superluminal radio source (Moffet et al. 1972); and the first quasar detected at the energies of $>1 \mathrm{GeV}$ with EGRET/ CGRO. The simultaneous variability in X-rays and $\gamma$-rays $(>100 \mathrm{MeV}$ ) suggests for the first time that they are approximately cospatial ( $\mathrm{M}^{\mathrm{c}} \mathrm{Hardy}$ 1996). The $\gamma$-ray flux varies from 1.28 to $28.710^{-6}$ photon $\mathrm{cm}^{-2} \mathrm{~s}^{-1}$ (Mukherjee et al. 1997). Two $\gamma$-ray flares were detected (see Kniffen et al. 1993; Hartman et al. 1996; McHardy 1996; Wehrle et al. 1998).

The 16-28 June 1991 flare showed: $F(>100 \mathrm{MeV})=$ $(2.8 \pm 0.4) 10^{-6}$ photon $\mathrm{cm}^{-2} \mathrm{~s}^{-1}$ with a photon spectral index $\alpha_{\gamma}=1.89 \pm 0.06$. A variation of a factor of 4 over 2 days was obtained.

The January-February 1996 flare showed (see McHardy 1996; Wehrle et al. 1998), $F(>100 \mathrm{MeV})=$ (11.0 \pm 1 .) $10^{-6}$ photon $\mathrm{cm}^{-2} \mathrm{~s}^{-1}$ with a photon spectral index $\alpha_{\gamma}=1.97 \pm 0.07$. During this flare, a variation of a factor of $4 \sim 5$ in a day is observed, $\Delta T=6$ hrs (Wehrle et al. 1998).

No obvious spectral index variation has been detected when the flux varied.

\subsubsection{PKS $1406-074$}

PKS $1406-074$ has been detected to vary with the $\gamma$-ray flux being in the range of 1.54 to $12.7610^{-6}$ photon $\mathrm{cm}^{-2}$ $\mathrm{s}^{-1}$ (Mukherjee et al. 1997). During its $\gamma$-ray flare, a flux of $F(>100 \mathrm{MeV})=(5.5 \pm 1.4) 10^{-6}$ photon $\mathrm{cm}^{-2} \mathrm{~s}^{-1}$ with a photon spectral index $\alpha_{\gamma}=2.04 \pm 0.15$ and a doubling timescale of shorter than 16 hours has been obtained (see Wagner et al. 1995).

\subsubsection{PKS $1622-297$}

For PKS $1622-297, z=0.815$, we have very little information in lower energy bands. But it is one of the most luminous objects in the $\gamma$-ray region. A peak flux of $(17 \pm 3) 10^{-6}$ photon $\mathrm{cm}^{-2} \mathrm{~s}^{-1}(E>100 \mathrm{MeV})$ and a flux increase by a factor of 2 in 9.7 hours were observed (Mattox et al. 1997).

\subsubsection{Q1633+382, 4C 38.41}

Quasar $1633+382, z=1.814$, is an LPQ $\left(P_{\text {opt }}=2.6 \%\right.$, Moore \& Stockman 1984). During 1992 November 17 - December 1 period, it was detected to show a flux of $F(>100 \mathrm{MeV})=(0.30 \pm 0.06) 10^{-6}$ photon $\mathrm{cm}^{-2} \mathrm{~s}^{-1}$ with a photon spectral index $\alpha_{\gamma}=1.87 \pm 0.07$. The flux varied by it a factor of 1.5 within $24 \mathrm{hr}, \Delta T_{\mathrm{D}}=16$ hrs, while the spectral index did not change. The $\gamma$-ray 
luminosity is at least two orders of magnitude larger than the maximum ever observed in any other band (see Mattox et al. 1993).

\subsubsection{B2 1652+399, Mkn 501}

Mkn 501, $z=0.033$, together with other two XBLs are three known $\mathrm{TeV} \gamma$-ray sources detected by the Whipple group (see Quinn et al. 1996; Catanese et al. 1997a; Samuelson et al. 1998; Kataoka et al. 1998).

During 1995 March-July period, Mkn 501 was observed to show a flux of $F(>300 \mathrm{GeV})=(8.1 \pm 1.4) 10^{-12}$ photon $\mathrm{cm}^{-2} \mathrm{~s}^{-1}$ with a photon spectral index $\alpha_{\gamma}=2.2$. A variation of a factor of 4 over one day is also detected, $\Delta T_{\mathrm{D}}=6 \mathrm{hrs}$. The upper limit corresponds to a flux of $F(>100 \mathrm{MeV})=1.510^{-7}$ photon $\mathrm{cm}^{-2} \mathrm{~s}^{-1}$ (see Quinn et al. 1996). During the 1996 multiwavelength campaign, Mkn 501 was detected with EGRET a flux of $F(>100$ $\mathrm{MeV})=(0.32 \pm .13) 10^{-6}$ photon $\mathrm{cm}^{-2} \mathrm{~s}^{-1}$ with a photon index of $1.6 \pm 0.5$ (see Kataoka et al. 1998). During 1997 April 9-19 observation, Catanese et al. (1997a) obtained $F(>300 \mathrm{GeV})=(40.5 \pm 9.6) 10^{-11}$ photon $\mathrm{cm}^{-2}$ $\mathrm{s}^{-1}, \alpha_{\gamma}=2.5$, the April 9-15 flux corresponds to a flux of $F(>100 \mathrm{MeV})<3.610^{-7}$ photon $\mathrm{cm}^{-2} \mathrm{~s}^{-1}$.

The TeV observations show that the spectrum softens when the source brightens.

\subsection{8. $2200+420$, BL Lacertae}

$2200+420$ is the prototype of BL Lacertae class. It is variable in all wavelengths (see Fan et al. 1998b, 1998c; Bloom et al. 1997; Böttcher \& Bloom 1998; Madejski et al. 1998). A 14-year period was found in the optical light curve (Fan et al. 1998b). During 1995 January 24 February 14, BL Lacertae showed a flux of $F(>100 \mathrm{MeV})$ $=(40 \pm 12) 10^{-8}$ photon $\mathrm{cm}^{-2} \mathrm{~s}^{-1}$ with a photon spectral index $\alpha_{\gamma}=2.2 \pm 0.3$, the up limit flux in higher energy is $F(>300 \mathrm{GeV})<0.5310^{-11}$ photon $\mathrm{cm}^{-2} \mathrm{~s}^{-1}$ (Catanese et al. 1997b). During 1997 January 15/22 observation period, it was detected a flux of $F(>100 \mathrm{MeV})=(171 \pm 42)$ $10^{-8}$ photon $\mathrm{cm}^{-2} \mathrm{~s}^{-1}$ with a photon spectral index $\alpha_{\gamma}=$ $1.68 \pm 0.16$ and a dramatic factor of 2.5 increase within a timescale of $8 \mathrm{hrs}, \Delta T_{\mathrm{D}}=3.2 \mathrm{hrs}$. Besides, simultaneous optical and $\gamma$-ray flares were observed ruling out external scattering models (see Bloom et al. 1997).

The observations from the object show that the spectrum of BL Lacertae hardens when the $\gamma$-ray flux increases.

\subsection{The central black hole mass and the Doppler factor}

The objects discussed here show variability time scale of hours to days. The variability could be directly related to shock processes in a jet, far from the accretion disk (we thank Dr. S.D. Bloom to point out this for us). If we take the variability timescale as the measurements of the size, $R$, of the emission region, then the $R$ in the jet obeys to the inequality,

$R \leq c \Delta T_{\mathrm{D}} \frac{\delta}{(1+z)} \mathrm{cm}$

where $c$ is the speed of light, $\delta$ the Doppler factor, $z$ the redshift of the source, and $\Delta T_{\mathrm{D}}$, in units of second, the doubling time scale.

For an object with a mass $M$, the Eddington limit gives (Frank et al. 1985)

$L_{\text {Edd. }} \approx 1.2610^{38}\left(\frac{M}{M_{\odot}}\right) \operatorname{erg~s}^{-1}$.

So, we have that the intrinsic luminosity, $L^{\text {in. }}$ of a source with a mass of $M$ should satisfy $L^{\text {in }} \leq L_{\text {Edd. }}$.

In the relativistic beaming frame, the observed luminosity is $L^{\mathrm{ob}}=\delta^{(4+\alpha)} L^{\mathrm{in}}, \alpha$ is the energy spectral index, which follows that

$L^{\text {ob. }} \leq \delta^{(4+\alpha)} L_{\text {Edd }}$

Ghisellini \& Madau (1996) obtained that the $\gamma$-rays are emitted within the BLR region, which is $10^{17-18} \mathrm{~cm}$ far from the central source. Hartman et al (1996) obtained that the $\gamma$-rays are produced at a distance of $\sim 100 R_{\mathrm{g}}$. Recently, Celotti \& Ghisellini (1998) argued that the $\gamma$-rays are from a region of some hundreds of Schwarzschild radii from the center. From our previous paper, a distance of $205 R_{\mathrm{g}}$ is obtained for the $\gamma$-rays from Mkn 421 . In the sense of the theory of accretion (Sunyaev 1975). When $R<200 R_{\mathrm{g}}$, the electrons in the accretion flow become ultrarelativistic. On the other hand, the mixture of relativistic electrons and nonrelativistic protons has an adiabatic index $\gamma<\frac{5}{3}$, with such an adiabatic index the transition to supersonic accretion regime is possible in the region $R<200 R_{\mathrm{g}}$ (Sunyaev 1975). So, the $200 R_{\mathrm{g}}$ is perhaps an important critical point. If we assume that the $\gamma$-rays are from this place then relations (1), (2), and (3) give

$$
\begin{aligned}
\frac{M}{M_{\odot}} & =510^{2} \frac{\delta}{1+z} \Delta T_{\mathrm{D}} \\
L^{\mathrm{ob}} & \leq 6.310^{40} \frac{\delta^{(5+\alpha)}}{(1+z)} \Delta T_{\mathrm{D}} \mathrm{erg} \mathrm{s}^{-1} .
\end{aligned}
$$

It is a common property of the EGRET-detected AGNs to show that their $\gamma$-ray flux is dominant over the flux in lower energy bands but this is not always the case (Mukherjee et al. 1997). For PKS 0528+134 and 3C 279, their $\gamma$-ray luminosity, $L_{\gamma}$, is $0.80 L_{\text {bol. }}$ and $0.5 L_{\text {bol. }}$, respectively (see Sambruna et al. 1997; Hartman et al. 1996). Because we consider the flare states of the selected objects, we can take the $\gamma$-ray luminosity to stand for half of the bolometrical luminosity approximately, i.e. $L_{\gamma} \sim 0.5 L_{\text {bol. }}$. So, we have

$L_{\gamma} \leq 3.1510^{40} \frac{\delta^{(5+\alpha)}}{(1+z)} \Delta T_{\mathrm{D}} \operatorname{erg~s}^{-1}$ 
which gives

$\delta \geq\left[\frac{L_{\gamma}(1+z)}{3.1510^{40} \operatorname{erg~s}^{-1} \Delta T_{\mathrm{D}}}\right]^{\left(\frac{1}{5+\alpha}\right)}$.

So, from the available $L_{\gamma}$ and $\Delta T_{\mathrm{D}}$, we can obtain the central black hole mass and the Doppler factor from relations (4) and (7) and $\alpha=\alpha_{\gamma}-1, \alpha_{\gamma}$ is the photon spectral index. They are shown in Table 1, in which Col. 1 gives the name; Col. 2, the redshift; Col. 3. the flux $F(>100 \mathrm{MeV})$ in units of $10^{-6}$ photon $\mathrm{cm}^{-2} \mathrm{~s}^{-1}, \sigma$ is the uncertainty; Col. 4, the photon spectral index, $\alpha_{\gamma}=2.0$ is adopted for $0537-441$ (see Fan et al. 1998a); Col. 5, the doubling time scale in units of hours; Col. $6, \gamma$-ray luminosity (assuming isotropic emission) in units of $10^{48} \mathrm{erg} \mathrm{s}^{-1}$; Col. 7, Doppler factor estimated from Eq. (7); Col. 8, the central black hole mass in units of $10^{7} M_{\odot}$; Col. 9 , the central black hole mass estimated from Dermer \& Gehrels (1996), thereafter D\&G, in units of $10^{7} M_{\odot}$; Col. 10, the mass estimated directly from Eddington limit in units of $10^{10} M_{\odot}$.

\section{Discussion}

\subsection{Mass}

From the high $\gamma$-ray luminosity (assuming isotropic emission) and Eddington-limit, one can derive the central black hole mass expression,

$M_{10} \geq \frac{L_{\mathrm{T}}}{1.2610^{48} \mathrm{erg} \mathrm{s}^{-1}}$

where, $L_{\mathrm{T}}$ is the bolometric luminosity for emission in the Thomson region, $M_{10}$ is the central black hole mass in units of $10^{10} M_{\odot}$. The derived masses are as high as $10^{11} M_{\odot}$ for some $\gamma$-ray loud blazars, PKS $0528+134$, PKS 1406 - 074, and PKS 1622 - 297 for instance (see Col. 10 in Table 1). However, for high energy $\gamma$-ray emission, Klein-Nishina effects must be considered. D\&G considered the effect and obtained an expression for the black hole mass, i.e. their Eq. (16b),

$M_{8}^{\mathrm{KN}} \geq \frac{3 \pi d_{\mathrm{L}}^{2}\left(m_{\mathrm{e}} c^{2}\right)}{2 \times 1.2610^{46} \mathrm{erg} \mathrm{s}^{-1}} \frac{F\left(\varepsilon_{\mathrm{l}}, \varepsilon_{\mathrm{u}}\right)}{1+z} \ln \left[2 \varepsilon_{\mathrm{l}}(1+z)\right]$

where $F\left(\varepsilon_{1}, \varepsilon_{\mathrm{u}}\right)$ is the integrated photon flux in units of $10^{-6}$ photon $\mathrm{cm}^{-2} \mathrm{~s}^{-1}$ between photon energies $\varepsilon_{\mathrm{l}}$ and $\varepsilon_{\mathrm{u}}$ in units of $0.511 \mathrm{MeV}$. For the objects considered here, $M_{7}^{\mathrm{KN}}$ is obtained and shown in Col. 9 in Table 1 . Table 1 shows that the masses obtained from our consideration and those estimated from the D\&G method are acceptably similar except for $1622-297$ and two low redshift BL Lac objects (Mkn 501 and BL Lacertae). For 1622 - 297 our value is about 7 times less than that estimated from the D\&G method. If we adopt the flux density $2.4510^{-6}$ photon $\mathrm{cm}^{-2} \mathrm{~s}^{-1}$ for $1622-297$ instead of the peak value as did Muhkerjee et al. (1997) and Fan et al. (1998a), then the isotropic luminosity is $3.8710^{48} \mathrm{erg} \mathrm{s}^{-1}$. This luminosity suggests that the Doppler factor and mass obtained from relations (4) and (7) are respectively 5.01 and $2.41 M_{7}$, and the mass estimated from the D\&G is then $3.61 M_{7}$. The two masses are quite similar in this case. For Mkn 501 and BL Lacertae, our value is much greater than that estimated from the D\&G method. For 3C 279, our results show that the estimated central black hole masses, $4.74 M_{7}$ and $3.43 M_{7}$ for 1991 and 1996 flares respectively, are almost the same, while the masses estimated from the D\&G method are $1.92 M_{7}$ and $7.53 M_{7}$ for 1991 and 1996 flares respectively. Table 1 (also see relation (9)) shows that the mass obtained from the D\&G method is sensitive to the flux, variable flux gives different mass for a source. The mass obtained from our method does not depend on the flux so sensitively. For 3C 2791991 and 1996 flares, masses obtained from our consideration are almost the same while those obtained from the D\&G method show a difference of more or less a factor of 4 . But our method depends on the timescales (see relation 4 ). Since we only considered the objects showing short timescales (hours) in the present paper, the masses obtained are in a range of $(1 \sim 7) 10^{7} M_{\odot}$.

To fit 3C 279 multiwavelength energy spectrum corresponding to $1991 \gamma$-ray flare, Hartman employed an accreting black hole of $10^{8} M_{\odot}$, our result of $4.7410^{7} M_{\odot}$ is similar to theirs.

\subsection{Beaming factors}

To explain the extremely high and violently variable luminosity of AGNs, the beaming model has been proposed. In this model, the Lorentz factor, $\Gamma$, and the viewing angle, $\theta$, are not measurable, but they can be obtained through the measurement of superluminal velocity, $\beta_{\text {app. }}$, and the determination of Doppler factor, $\delta$, which are related with the two unmeasurable parameters, $\Gamma$ and $\theta$, in the forms: $\beta_{\text {app }}=\frac{\beta_{\text {in } \sin \theta}}{\left(1-\beta_{\text {in }} \cos \theta\right)}, \Gamma=\frac{1}{\sqrt{1-\beta_{\text {in }}^{2}}}$, and $\delta=\left(\Gamma\left(1-\beta_{\text {in }} \cos \theta\right)\right)^{-1}$. So, $\Gamma$ and $\theta$ can be obtained from the following relations:

$\Gamma=\frac{\beta_{\mathrm{app}}^{2}+\delta^{2}+1}{2 \delta}$

$\theta=\tan ^{-1}\left(\frac{2 \beta_{\mathrm{app}}}{\beta_{\mathrm{app}}^{2}+\delta^{2}-1}\right)$

From our previous work (Fan et al. 1996), we can get superluminal velocities for 3C 279 and BL Lacertae. When the superluminal velocities and the derived Doppler factor are substituted to the above two relations. we found that: For $3 \mathrm{C} 279, \Gamma=2.4-14.4$ and $\theta=10^{\circ} .9-15.6$ for $\delta=3.37$; and $\Gamma=2.95-11.20$ and $\theta=8.45-9.7$ for $\delta=4.89$. For BL Lacertae $\Gamma=2{ }^{\circ}-4^{\circ}$. and $\theta=25^{\circ}$. $-29{ }^{\circ}$. .

To let the optical depth $\left(\tau_{\gamma \gamma}\right)$ be less than unity, Doppler factor in $\gamma$-ray region has been obtained for some objects by other authors. $\delta \geq 7.6$ for Q1633+382 (Mattox et al. 1993); $\delta \geq 6.3-8.5$ for 3C 2791996 flare (Wehrle 
Table 1. Mass and Doppler factor for $\gamma$-ray loud blazars

\begin{tabular}{lccccccccc}
\hline Name & $z$ & $F(\sigma)$ & $\alpha_{\gamma}$ & $\Delta T_{\mathrm{D}}$ & $L_{48}$ & $\delta$ & $M_{7}$ & $M_{7}^{\mathrm{KN}}$ & $M_{10}^{\mathrm{T}}$ \\
$(1)$ & $(2)$ & $(3)$ & $(4)$ & $(5)$ & $(6)$ & $(7)$ & $(8)$ & $(9)$ & $(10)$ \\
\hline $0528+134$ & 2.07 & $3.08(0.35)$ & 2.21 & 24. & 18.4 & 4.96 & 6.97 & 22.98 & 14.6 \\
$0537-441$ & 0.894 & $2.0(0.4)$ & 2.0 & 16. & 3.01 & 3.83 & 5.82 & 3.48 & 2.39 \\
$1253-055$ & 0.537 & $2.8(0.4)$ & 2.02 & 12. & 1.34 & 3.37 & 4.74 & 1.92 & 1.06 \\
$1253-055$ & 0.538 & $11 .(1)$. & 1.97 & 6. & 5.75 & 4.89 & 3.43 & 7.53 & 4.56 \\
$1406-074$ & 1.494 & $5.5(1.6)$ & 2.04 & 16.21 & 23.7 & 5.57 & 6.52 & 23.6 & 18.81 \\
$1622-297$ & 0.815 & $17 .(3)$. & 1.87 & 4.85 & 26.9 & 6.97 & 3.35 & 25. & 21.35 \\
$1633+382$ & 1.814 & $0.96(0.08)$ & 1.86 & 16. & 9.72 & 5.16 & 5.28 & 5.74 & 7.71 \\
$1652+399$ & 0.033 & $<0.36$ & 2.5 & 6. & 0.0003 & 0.89 & 0.94 & 0.001 & 0.00026 \\
$2200+420$ & 0.07 & $1.71(0.42)$ & 1.68 & 3.2 & 0.019 & 2.04 & 1.09 & 0.02 & 0.015 \\
\hline
\end{tabular}

et al. 1998) and $\delta \geq 3.9$ for 3C 2791991 flare (Mattox et al. 1993), $\delta \sim 5$ is also obtained by Henri et al. (1993); $\delta \geq 6.6 \sim 8.1$ for PKS $1622-297$ (Mattox et al. 1997). Our results in Table 1 are consistent with those results.

\subsection{Summary}

In this paper, the central mass and Doppler factor are obtained for $8 \gamma$-ray loud blazars with available short $\gamma$-ray timescales. The mass obtained from relation (4) in the present paper is compared with that obtained from the D\&G method, the masses obtained from two methods are similar for 5 out of 8 objects. Our method is not as sensitive to the flux as the D\&G method in estimating the central black hole mass. The masses obtained here are in a range of $(1 \sim 7) \quad 10^{7} M_{\odot}$, which stems from the fact that the time scales considered here are in a range of 3.2 to 24 hours. For $3 \mathrm{C} 279$, the masses obtained from the two flares are almost the same. For 3C 279 and BL Lacertae, the Lorentz factor and viewing angle are estimated.

Acknowledgements. The authors thank the referee Dr. S.D. Bloom for his comments and suggestions. J.H.F. thanks Drs. Böttcher, Dermer, Carter-Lewis, Catanese, Ghisellini, Kataoka, and Wagner for their sending him their publications and information and the Education fund of Guangzhou City for support. This work is supported by the National Pan Deng Project of China.

\section{References}

Blandford R.D., Levinson A., 1995, ApJ 441, 79

Bloom S.D., Bertsch D.L., Hartman R.C., et al., 1997, ApJ 490, L145

Bloom S.D., Marscher A.P., 1996, ApJ 461, 657

Bloom S.D., Marscher A.P., 1992, in: the Compton Observatory Science Workshop, Shrader C.R., Gehrels N., Dennis B. (eds.), p. 339

Bloom S.D., Marscher A.P., 1993, in: AIP Conf. Proc. 280, Proc. Compton Symp., Friedlander M., Gehrels N., Macomb D.J. (eds.), p. 578

Böttcher M., Bloom S.D., 1998, ApJ (submitted)
Böttcher M., Collmar W., 1998, A\&A 327, L57

Catanese M., Bradbury S.M., Breslin A.C., et al., 1997a, ApJL 487, L143

Catanese M., Akerlof C.W., Biller S.D., et al., 1997b, ApJ 480, 562

Catanese M., Akerlof C.W., Bradbury S.M., et al., 1998, ApJ 501,616

Celotti A., Ghisellini G., 1998, BL Lac Phenomena, June 22-26, Turku, Funland

Cheng K.S., Ding W.K.Y., 1994, A\&A 288, 97

Collmar W., Bennett K., Bloemen H., et al., 1997, A\&A 328 , 33

Coppi P.S., Kartje J.F., Königl A., 1993, in AIP Conf. Proc. 280, Proc. Compton Symp., Friedlander M., Gehrels N., Macomb D.J. (eds.), p. 559

Dermer C.D., Gehrels N., 1995, ApJ 447, 103

Dermer C.D., Schlickeiser R., Mastichiadis A., 1992, A\&A 256, L27

Dondi L., Ghisellini G., 1995, MNRAS 273, 583

Fan J.H., Lin R.G., 1998, ApJS (accepted)

Fan J.H., Adam G., Xie G.Z., et al., 1998a, A\&A 338, 27

Fan J.H., Xie G.Z., Pecontal E., et al., 1998b, ApJ (in press)

Fan J.H., Xie G.Z., Lin R.G., Qin Y.P., 1998c, A\&AS 132

Fan J.H., Cheng K.S., Zhang L., Liu C.H., 1997, A\&A 327, 947

Fan J.H., 1997, Ap\&SS 246, 119

Fan J.H., Xie G.Z., Wen S.L., 1996, A\&AS 116, 409

Frank J., King A.R., Rain D.J., Accretion Power in Astrophysics. Cambridge: Cambridge Uni. 1985

Ghisellini G., Madau P., 1996, MNRAS 280, 67

Hartman R.C., Webb J.R., Marscher A.P., et al., 1996, ApJ 461,698

Hartman R.C., 1996, ASP Conf. Ser. 110, 33

Henri G., Pelletier G., Roland J., 1993, ApJ 404, L41

Hunter S.D., Bertsch D.L., Dingus B.L., et al., 1993, ApJ 409, 134

Kataoka J., et al., 1998, ApJ (submitted)

Kniffen D.A., Bertsch D.L., Fichtel C.E., et al., 1993, ApJ 411, 133

Madejski G., et al., 1998, ApJ (submitted)

Mannheim K., 1993, Phys. Rev. D48, 2408

Mannheim K., Biermann P.L., 1992, A\&A 253, L21

Maraschi L., Ghisellini G., Celotti A., 1992, ApJ 397, L5

Marscher A.P., Travis J.P., 1996, A\&AS 120, 537

Mattox J.R., Bertsch D.L., Chiang J., et al., 1993, ApJ 410, 609

Mattox J.R., Wagner S.J., 1996, ASP Conf. Ser. 110, 352 
Mattox J.R., Wagner S.J., Malkan M., et al., 1997, ApJ 476, 692

McNaron-Brown K., Johnston W.N., Jung G.V., et al., 1995, ApJ 451, 575

McHardy I., 1996, ASP Conf. Ser. 110, 293

Mücke A., Pohl M., Reich P., et al., 1997, A\&A 320, 33

Moore R.L., Stockman H.S., 1984, ApJ 279, 465

Moffet A.T., Gubbay, J. Robertson D.S., Legg A.J., 1972, in IAU Symp. 44. Evans D.S. (eds.), p. 28

Mukherjee R., Bertsch D.L., Bloom S.D., et al., 1997, ApJ 490, 116

Mukherjee R., Dingus B.L., Gear W.K., et al., 1996, ApJ 470, 831

Punch M., Akerlof C.W., Cawley M.F., et al., Nat 358, 477

Quinn J., Akerlof C.W., Biller S., et al., 1996, ApJ 456, L83

Samuelson F.W., Biller S.D., Bond I.H., et al., 1998, ApJ 501, L17

Sambruna R., Urry C.M., Maraschi L., et al., 1997, ApJ 474, 639
Sikora M., Begelman M.C., Rees M.J., 1994, ApJ 421, 153

Sunyaev R.A., 1975, in: Proceedings of the International School of Physics ENRICO FFERMI, Course LXV, Giacconi R., Ruffini R. (eds.), p. 697

Surpi G.C., Romero G.E. Vucetich H., 1996, RMxAA 32, 153 von Montigny C., Aller H., Aller M., et al., 1997, ApJ 483, 161 von Montigny C., Bertsch D.L., Chiang J., et al., 1995, ApJ 440,525

Wagner S.J., Mattox J.R., Hopp U., 1995, ApJ 454, L97

Wagner S.J., von Montigny C., Herter M., 1997, 4th Compton Symposium, Dermer C.D., et al., (eds.), AIP 410, p. 1457

Wehrle A.E., Pian E., Urry C.M., et al., 1998, ApJ 497, 178

Xie G.Z., Bai J.M., Zhang X., Fan J.H., 1998, A\&A 334, L29

Xie G.Z., Zhang Y.H., Fan J.H., 1997, ApJ 477, 114

Zdziarski A.A., Krolik J.H., 1993, ApJ 409, L33

Zhou Y.Y., Lu Y.J., Wang T.G., et al., 1997, ApJ 484L, 47 